\title{
Comparative Analysis of Cesium Chloride- and lodixanol-Based Purification of Recombinant Adeno-Associated Viral Vectors for Preclinical Applications
}

\author{
Benjamin Strobel, ${ }^{1, *}$ Felix D. Miller, ${ }^{1, *}$ Wolfgang Rist, ${ }^{2}$ and Thorsten Lamla, ${ }^{1, \dagger}$ \\ ${ }^{1}$ Target Discovery Research and ${ }^{2}$ Drug Discovery Support, Boehringer Ingelheim Pharma GmbH \& Co. KG, Biberach an der Riss, Germany. \\ * These authors contributed equally to this work.
}

Cesium chloride ( $\mathrm{CsCl}$ )- and iodixanol-based density gradients represent the core step in most protocols for serotype-independent adeno-associated virus (AAV) purification established to date. However, despite controversial reports about the purity and bioactivity of AAV vectors derived from each of these protocols, systematic comparisons of state-of-the-art variants of these methods are sparse. To define exact conditions for such a comparison, we first fractionated both gradients to analyze the distribution of intact, bioactive AAVs and contaminants, respectively. Moreover, we tested four different polishing methods (ultrafiltration, size-exclusion chromatography, hollow-fiber tangential flow filtration, and polyethylene glycol precipitation) implemented after the iodixanol gradient for their ability to deplete iodixanol and protein contaminations. Last, we conducted a side-by-side comparison of the $\mathrm{CsCl}$ and iodixanol/ultrafiltration protocol. Our results demonstrate that iodixanol-purified AAV preparations show higher vector purity but harbor more $(\sim 20 \%)$ empty particles as compared with CsCl-purified vectors $(<1 \%)$. Using mass spectrometry, we analyzed prominent protein impurities in the AAV vector product, thereby identifying known and new, possibly AAV-interacting proteins as major contaminants. Thus, our study not only provides a helpful guide for the many laboratories entering the AAV field, but also builds a basis for further investigation of cellular processes involved in AAV vector assembly and trafficking.

\section{INTRODUCTION}

RECOMBINANT ADENO-ASSOCIATED VIRAL (AAV) vectors have evolved as one of the most heavily used vector tools to study gene function both in vitro and in vivo. Besides the fact that $\mathrm{AAVs}$ are regarded as nonpathogenic, one major advantage of $\mathrm{AAVs}$ is the lack of inflammation after vector application in vivo, ${ }^{1}$ thereby lowering the risk of altering experimental readouts by vector-induced immunogenic effects. In this regard, high purity of vector preparations is a key goal of AAV manufacturing both for research and clinical applications. For the serotype-independent purification of AAV vectors produced in HEK-293 or SF9 cells, the two most widely established protocols for preclinical applications are based on ultracentrifugation using either a continuous cesium chlo- ride $(\mathrm{CsCl})$ density gradient ${ }^{2}$ or an iodixanol step density gradient. ${ }^{3}$ While the $\mathrm{CsCl}$ protocol contains multiple purification steps (cell lysis, precipitation of DNA and proteins, ultracentrifugation, dialysis, concentration; see Fig. 1), rendering it a rather time-consuming ( $\sim 3.5$ days) protocol, the iodixanol protocol requires only cell lysis, $2 \mathrm{hr}$ of ultracentrifugation, and concentration, allowing for vector purification in 1 day (Fig. 1).

Although both protocols are widely used, analyses of vector purity and bioactivity after purification by each of these methods are sparse. ${ }^{3,4}$ Surprisingly, except from the comparison of iodixanol-based purification to two rounds of $\mathrm{CsCl}$ centrifugation more than 15 years ago, ${ }^{3}$ no direct comparison of current variants of these protocols has been published so far.

\footnotetext{
${ }^{\dagger}$ Correspondence: Dr. Thorsten Lamla, Birkendorfer Str. 65, 88397 Biberach an der Riss, Germany. E-mail: thorsten.lamla@boehringer-ingelheim.com
}

(C) Benjamin Strobel et al. 2015; Published by Mary Ann Liebert, Inc. This Open Access article is distributed under the terms of the Creative Commons Attribution Noncommercial License (http://creativecommons.org/licenses/by-nc/4.0/) which permits any noncommercial use, distribution, and reproduction in any medium, provided the original author(s) and the source are credited. 


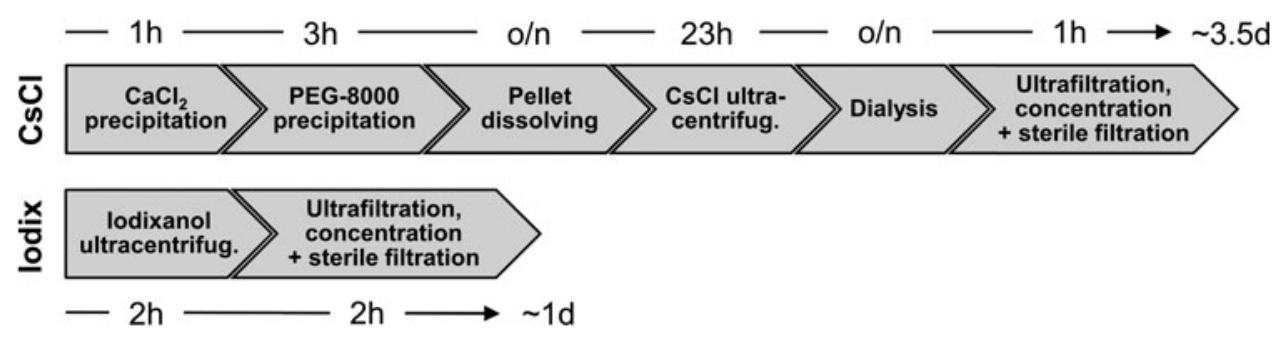

Figure 1. Process steps and duration of $\mathrm{CsCl}$ - and iodixanol-based adeno-associated virus (AAV) purification. After cell lysis by three repeated freeze-thaw cycles, the AAV-containing lysate is purified by either a $\mathrm{CsCl}$ - or iodixanol-based purification process. $\mathrm{CsCl}$ : Nucleic acids and proteins are pelleted by $\mathrm{CaCl}_{2}$ and PEG-8000 precipitation, respectively. After protein pellet dissolving overnight (o/n) and subsequent $\mathrm{CsCl}$ density gradient ultracentrifugation, CsCl is removed from the target fractions by repeated dialysis cycles. The AAV suspension is finally concentrated by ultrafiltration and sterile filtered. lodixanol: The cell lysate is applied to iodixanol density gradient ultracentrifugation. lodixanol is removed by three repeated ultrafiltration/concentration steps and the AAV suspension is finally sterile filtered.

Therefore, we carried out a systematic side-by-side analysis of the CsCl- and iodixanol-based protocols.

To clearly define the exact conditions for our comparison, we first conducted an in-depth analysis of both density gradients. To this end, the gradients were fractionated and analyzed for the distribution of intact, bioactive AAV particles and unwanted contaminations such as plasmid/genomic DNA and empty vector particles. Moreover, to optimize the iodixanol-based protocol we compared ultrafiltration, size-exclusion chromatography (SEC), hollow-fiber tangential flow filtration (HF-TFF), and polyethylene glycol (PEG) precipitation with respect to their purification efficiency as a polishing step after the iodixanol gradient. After having established specific criteria for both the $\mathrm{CsCl}$ and iodixanol protocols, AAVs were purified by both methods in a side-by-side experiment and subsequently compared for purity and in vitro bioactivity. Moreover, we applied transmission electron microscopy (TEM) to assess the full-to-empty particle ratio, and mass spectrometry (MS) to identify contaminating proteins in the final product preparations.

\section{MATERIALS AND METHODS}

\section{AAV production and cell lysis}

HEK-293h cells were cultivated in Dulbecco's modified Eagle's medium (DMEM)-GlutaMAX (Life Technologies, Karlsruhe, Germany) supplemented with $10 \%$ fetal calf serum (FCS). Three days before transfection, the cells were seeded in 15-cm tissue culture plates (Sarstedt, Nümbrecht, Germany) to reach 70-80\% confluence on the day of transfection. For transfection, $0.5 \mu \mathrm{g}$ of total DNA per centimeter squared of culture area was mixed with a 1/10th culture volume of $300 \mathrm{mM} \mathrm{CaCl} 2$, whereby pDP8.ape (PlasmidFactory, Bielefeld, Germany) and a pAAVCMV-GFP plasmid were used in an equimolar ratio. This mix was then added dropwise to an equal volume of $2 \times$ HEPES-buffered saline (HBS; $50 \mathrm{mM}$
HEPES, $280 \mathrm{~m} M \mathrm{NaCl}, 1.5 \mathrm{~m} M \mathrm{Na}_{2} \mathrm{HPO}_{4}$ ), incubated for $2 \mathrm{~min}$ at room temperature, and added to the cells. After 5-6 hr of incubation, the culture medium was replaced with fresh medium. The transfected cells were grown at $37^{\circ} \mathrm{C}$ for a total of $72 \mathrm{hr}$. Cells were detached by the addition of EDTA to a final concentration of $6.25 \mathrm{mM}$ and pelleted by centrifugation at $1000 \times \mathrm{g}$ for $10 \mathrm{~min}$ at room temperature. The cells were then resuspended in lysis buffer ( $50 \mathrm{~m} M$ Tris, $150 \mathrm{~m} M \mathrm{NaCl}, 2 \mathrm{~m} M \mathrm{MgCl}_{2}, \mathrm{pH}$ 8.5). For $\mathrm{CsCl}$ purification, the cells of ten $15-\mathrm{cm}$ plates were dissolved in $30 \mathrm{ml}$ of lysis buffer. For one iodixanol gradient, the desired amount of cells (up to 40 plates) was dissolved in $8 \mathrm{ml}$ of lysis buffer. Cells were then lysed by three freeze-thaw cycles using liquid nitrogen and a $37^{\circ} \mathrm{C}$ water bath, respectively. For each initially transfected plate, 100 units of Benzonase nuclease (Merck, Darmstadt, Germany) was added to the mix and incubated for $1 \mathrm{hr}$ at $37^{\circ} \mathrm{C}$. After pelleting cell debris for $15 \mathrm{~min}$ at $2500 \times \mathrm{g}$, the supernatant was used for further purification.

\section{CsCl-based purification protocol}

$\mathrm{CaCl}_{2}$ was added to the supernatant obtained after cell lysis and Benzonase treatment (described previously) at a final concentration of $25 \mathrm{~m} M$. After incubating the mix for $1 \mathrm{hr}$ on ice, it was centrifuged for $15 \mathrm{~min}$ at $2500 \times \mathrm{g}$ and $4^{\circ} \mathrm{C}$, and the pellet was discarded. PEG-8000 (40\%) was added to the supernatant to a final concentration of $8 \%$ and the mix was incubated on ice for $3 \mathrm{hr}$. After centrifugation for $30 \mathrm{~min}$ at $2500 \times \mathrm{g}$ and $4^{\circ} \mathrm{C}$, the pellet was resuspended in resuspension buffer $(50 \mathrm{~m} M$ HEPES, $150 \mathrm{~m} M \mathrm{NaCl}, 25 \mathrm{~m} M$ EDTA, $\mathrm{pH}$ 7.4) and dissolved overnight at $4^{\circ} \mathrm{C}$ on a tube rotator. The suspension was then centrifuged for $30 \mathrm{~min}$ at $4^{\circ} \mathrm{C}$ and $2500 \times g$ and $3.149 M \mathrm{CsCl}$ was added to the supernatant (corresponding to a refractive index [RI] of 1.3710). Using 39-ml Quick-Seal tubes and a $70 \mathrm{Ti}$ rotor (both from Beckman Coulter, Brea, CA), the mix 
was ultracentrifuged for $23 \mathrm{hr}$ at $63,000 \mathrm{rpm}$ and $21^{\circ} \mathrm{C}$. Each tube was then punctured at the bottom, using a 20-gauge needle, and 1-ml fractions were collected. All fractions within an RI range of 1.3758 to 1.3703 were pooled and applied to five rounds of dialysis against 2 liters of phosphate-buffered saline (PBS), using Slide-A-Lyzer MWCO (molecular weight cutoff) 20,000 dialysis cassettes (Thermo Fisher Scientific/Pierce, Waltham, MA). The dialyzed product was finally concentrated with Amicon Ultra-15 centrifugal filter units (MWCO, $100 \mathrm{kDa}$; Merck Millipore, Billerica, MA). After addition of glycerol to a final concentration of $10 \%$, the product was sterile filtered with Ultrafree-CL filter tubes (Merck Millipore), aliquoted, and stored at $-80^{\circ} \mathrm{C}$.

\section{lodixanol-based purification protocol}

An iodixanol step density gradient was prepared essentially as described previously ${ }^{3}$ with slight modifications: Briefly, 60\% iodixanol (OptiPrep; Sigma-Aldrich, St. Louis, MO) was diluted to 15, 25, 40 , and $58 \%$ in PBS-MK $\left(1 \times \mathrm{PBS}, 1 \mathrm{mM} \mathrm{MgCl}_{2}\right.$, $2.5 \mathrm{~m} M \mathrm{KCl}) . \mathrm{NaCl}$ was added to the $15 \%$ phase at 1 $M$ final concentration. A $1.5-\mu \mathrm{l}$ volume of $0.5 \%$ phenol red was added per milliliter to the 15 and $25 \%$ iodixanol solutions and $0.5 \mu \mathrm{l}$ was added to the $58 \%$ phase to facilitate easier distinguishing of the phase boundaries within the gradient. Eight milliliters of $15 \%, 6 \mathrm{ml}$ of $25 \%, 8 \mathrm{ml}$ of $40 \%$, and $5 \mathrm{ml}$ of $58 \%$ iodixanol solution were successively underlaid in 39-ml Quick-Seal tubes (Beckman Coulter). The processed cell lysate (described previously) was then gently overlaid onto the gradient and filled up with lysis buffer when necessary. After centrifugation in a $70 \mathrm{Ti}$ rotor for $2 \mathrm{hr}$ at $63,000 \mathrm{rpm}$ and $18^{\circ} \mathrm{C}$, the tube was punctured at the bottom, using a 16-gauge needle, and 1-ml fractions were collected. For AAV collection, the first $5 \mathrm{ml}$ (corresponding to the 58\% phase) was discarded, and the fractions obtained from the $40 \%$ phase were analyzed by measuring absorbance at 20 -fold dilution at $340 \mathrm{~nm}$ to identify the main contaminating protein peak, as previously described. ${ }^{5}$ All fractions below this peak were pooled. PBS was added to the pool to reach a total volume of $15 \mathrm{ml}$ and ultrafiltered/concentrated, using Amicon Ultra-15 centrifugal filter units (MWCO, $100 \mathrm{kDa}$; Merck Millipore). After concentration to $\sim 1 \mathrm{ml}$, the retentate was filled up to $15 \mathrm{ml}$ and concentrated again. This process was repeated until an arithmetical value of $<0.1 \%$ iodixanol concentration was reached (usually three cycles). Glycerol was added to the preparation at a final concentration of $10 \%$. After sterile filtration with Ultrafree-CL filter tubes (Merck Millipore), the product was aliquoted and stored at $-80^{\circ} \mathrm{C}$.

\section{Size-exclusion chromatography}

A PD-10 desalting column filled with Sephadex G-25 medium (GE Healthcare, Piscataway, NJ) was equilibrated with four 2.5-ml volumes of PBS. The pooled AAV target fractions harvested from an iodixanol density gradient were applied to the column and the flow-through was discarded. AAVs were eluted by adding $3.5 \mathrm{ml}$ of PBS. The salt fraction was eluted for analytical purposes by addition of another $3.5 \mathrm{ml}$ of PBS. The AAV eluate was finally concentrated with Amicon Ultra-15 centrifugal filter units (MWCO, $100 \mathrm{kDa}$; Merck Millipore).

\section{Hollow-fiber tangential flow filtration}

Using a MicroKros hollow-fiber module equipped with a CO2-E100-05-S MWCO $100 \mathrm{kDa}$ membrane (Spectrum Laboratories, Los Angeles, CA), the iodixanol gradient-derived AAV pool was concentrated to $\sim 1 \mathrm{ml}$, before $10 \mathrm{ml}$ of $\mathrm{PBS}-10 \%$ glycerol was added to the retentate. Three rounds of this concentration/rebuffering step were carried out in total.

\section{PEG precipitation}

$\mathrm{CaCl}_{2}$ and PEG-8000 precipitation were conducted as described in the section CsCl-Based Purification Protocol to further purify iodixanol gradient-derived AAVs. In contrast to the previous description, the PEG-precipitated pellet was resuspended (without incubation overnight) and immediately applied to ultrafiltration using Amicon Ultra-15 centrifugal filter units (MWCO, $100 \mathrm{kDa}$; Merck Millipore). After concentration to $\sim 1 \mathrm{ml}$, the retentate was filled up to $15 \mathrm{ml}$ with $\mathrm{PBS}$ and concentrated again. Three rounds of this concentration/rebuffering step were carried out in total.

\section{Density gradient fraction analysis}

AAV vector genomes, plasmid DNA, and genomic DNA were detected by qPCR and primer-probe sets specific for, respectively, the CMV promoter sequence contained within the GFP transgene cassette, the ampicillin resistance gene present in the transgene plasmid backbone, and the RNA polymerase II gene (POLR2A) that served as a surrogate for genomic DNA. Plasmid and genomic DNAs were measured directly in diluted gradient fraction samples. For the detection of AAV vector genomes, viral DNA was isolated with a ViralXpress nucleic acid extraction kit (Merck Millipore). qPCR was conducted with $2 \times$ QuantiFast probe PCR master mix (Qiagen, Hilden, Germany) and the ABI PRISM 7900HT system (Applied Biosystems, Carlsbad, CA). To detect AAV8 capsids, an AAV8 titration 
ELISA kit (Progen Biotechnik, Heidelberg, Germany) was used. For the detection of bioactive AAVs, HEK-293 cells were plated at $70 \%$ confluence and treated with $15 \mu \mathrm{l}$ of each gradient fraction, each of which was previously dialyzed to remove cytotoxic $\mathrm{CsCl}$. Forty-eight hours after transduction, cells were trypsinized, washed, and resuspended in PBS$10 \%$ FCS. The cells were analyzed for GFP expression, using a BD FACSCanto flow cytometer (BD Biosciences, San Jose, CA).

\section{Sodium dodecyl sulfate-polyacrylamide gel electrophoresis}

For sodium dodecyl sulfate-polyacrylamide gel electrophoresis (SDS-PAGE) analysis, samples were denatured in lane marker reducing sample buffer (Life Technologies, Karlsruhe, Germany) at $95^{\circ} \mathrm{C}$ before being loaded onto NuPAGE 4-12\% Bis-Tris minigels (Life Technologies). After electrophoresis, gels were either silver-stained, using a PageSilver silver staining kit or PageBlue protein staining kit (both from Thermo Fisher Scientific/Fermentas, Waltham, MA).

\section{Transmission electron microscopy}

Electron microscopy grids were loaded with $5 \mu \mathrm{l}$ of AAV sample and stained with $2 \%$ phosphotungstic acid, $\mathrm{pH}$ 7.4. Microscopy was carried out with an EM 912 AB transmission electron microscope (Zeiss SMT, Oberkochen, Germany). The empty particle content of AAV samples was determined by counting full and empty AAV particles in the micrographs.

\section{Liquid chromatography-tandem mass spectrometry measurements}

Protein bands were excised and in-gel digested with modified porcine trypsin (Promega, Madison, WI) as described. ${ }^{6}$ Nanoscale liquid chromatography-tandem mass spectrometry (nanoLC-MS/MS) analysis was performed with a nanoAcquity UPLC connected to a Q-TOF (quadrupole time of flight) SYNAPT G2-Si mass spectrometer (Waters, Manchester, UK) operating in data-dependent mode, automatically switching between MS and MS2. ${ }^{7}$ A reference lockspray signal of Glu-fibrinopeptide was acquired for internal calibration. The raw data were processed with PLGS version 3.0.2 and searched against the UniProt database, using the Mascot search engine (Matrix Science, London, UK). All hits were manually verified on the basis of accepted rules for peptide fragmentation. ${ }^{8}$

For semiquantitative determination of iodixanol, full-scan MS spectra were acquired in resolution mode. Peak areas of extracted ion chromatograms were determined with MassLynx version 4.1 and a mass extraction window of $5 \mathrm{ppm}$, and samples were compared with iodixanol-spiked buffer.

\section{RESULTS}

\section{Analysis of fractionated $\mathrm{CsCl}$ density gradient}

AAV8-GFP vectors were produced in HEK-293 cells, as described in detail in Materials and Methods. Seventy-two hours after transfection, the cells of 20 transfected 15 -cm plates were harvested and lysed by three repeated freeze-thaw cycles and the resulting lysate was digested with Benzonase for $1 \mathrm{hr}$ at $37^{\circ} \mathrm{C}$. After pelleting residual DNA with $\mathrm{CaCl}_{2}$, proteins including AAVs were precipitated with PEG- 8000 and the resulting pellet was redissolved overnight. After addition of cesium chloride and adjustment of the refractive index (RI), the density gradient was ultracentrifuged for $23 \mathrm{hr}$. One-milliliter fractions were collected from the bottom of the tube for subsequent analyses.

qPCR analysis revealed that $\mathrm{AAV}$ vector genomes (VG) were detectable mainly in fractions 13-20 (Fig. 2a), corresponding to an RI range of 1.36931.3772 (Supplementary Fig. S1; supplementary data are available online at http://online.liebertpub.com/ hgtb). Plasmid DNA was present in fractions 1-5, 11-19, and 26-27, whereas genomic DNA was measurable only in fractions 1-4, 26, and 27 (Fig. 2a). As expected, AAV8 capsids were present mainly in fractions 17-20 (Fig. 2b). However, a small but distinct peak was also evident in fraction 23 (Fig. $2 b)$. Although complicated by impurities in this fraction, TEM analysis confirmed the assumption that this peak represents empty particles (Supplementary Fig. S2). To identify bioactive AAV particles, we next added $15 \mu \mathrm{l}$ of each fraction to HEK-293 cells and analyzed GFP expression 2 days later by flow cytometry. Our results show that GFP-positive cells could be detected mainly in fractions 13-20 (Fig. 2b), with a peak in fraction 17. Finally, to assess the extent of protein contaminants, we analyzed fractions 12-21 by SDS-PAGE. Whereas the AAV capsid proteins VP1 $(87 \mathrm{kDa}), \mathrm{VP} 2(73 \mathrm{kDa})$, and VP3 $(62 \mathrm{kDa})$ were visible mainly in fractions 15-20, relatively strong background and several distinct protein contaminants were visible in all fractions, with a clear trend toward more contamination in the upper fractions, particularly fractions 20 and 21 (Fig. 2c). Taken together, to obtain bioactive AAV particles with the lowest level of impurities possible, fractions 14 (RI, 1.3758) to 19 (RI, 1.3703) should be isolated. 




Figure 2. Analysis of the fractionated $\mathrm{CsCl}$ density gradient. One-milliliter fractions were collected from the bottom of the ultracentrifugation tube and analyzed for bioactive AAV particles and contaminants. (a) AAV vector genomes (VG), contaminating plasmid DNA, and genomic DNA were detected by qPCR analysis using primers specific for the cytomegalovirus (CMV) promoter sequence present in the vector genome, the ampicillin resistance gene present in the plasmid backbone, and the RNA polymerase II gene, respectively. (b) AAV8 capsid epitopes were detected by ELISA measurements. For the detection of bioactive AAV particles, HEK293 cells were treated with $15 \mu \mathrm{l}$ of each dialyzed fraction and GFP-positive cells were detected by flow cytometry $48 \mathrm{hr}$ posttransduction. (c) Silver-stained SDSpolyacrylamide gel of gradient fractions 12-21. The volume corresponding to $5 \times 10^{9} \mathrm{VG}$ in fraction 17 was calculated and equal volumes of the other fractions were loaded accordingly. M, marker lane. Asterisks indicate VP1 (87 kDa), VP2 (73 kDa), and VP3 (62 kDa). Color image available online at www.liebertpub.com/hgtb

\section{Analysis of fractionated iodixanol density gradient}

Analogous to the fractionation of the $\mathrm{CsCl}$ gradient, we next studied AAV distribution in the iodixanol step gradient. Again, AAV8-GFP vectors were produced and the cells were lysed $72 \mathrm{hr}$ after transfection. After pelleting cell debris, the lysate was directly applied on top of a previously layered step gradient, consisting of $8 \mathrm{ml}$ of a $15 \%, 6 \mathrm{ml}$ of a $25 \%, 8 \mathrm{ml}$ of a $40 \%$, and $5 \mathrm{ml}$ of a $58 \%$ iodixanol solution (from top to bottom). After $2 \mathrm{hr}$ of ultracentrifugation, 1-ml fractions were collected from the bottom and analyzed using the same readouts as described previously for the $\mathrm{CsCl}$ gradient.

Our results show that AAV vector genomes could be detected mainly in fractions $6-11$, with additional CMV-positive sequences also measured up to fraction 27 (Fig. 3a). Plasmid DNA was measured mainly in fractions 5-7 and 12-27 (Fig. 3a), whereas genomic DNA was present solely in fractions 12-27. AAV8 capsids were detected in fractions 5-13 (Fig. 3b). Notably, in contrast to the $\mathrm{CsCl}$ gradient, where two peaks were obtained, here just one main peak around fraction 7 was present; however, this peak showed a "shoulder" in fractions 12 and 13 (Fig. 3b). We next analyzed the fractions for bioactive AAVs and obtained GFPpositive HEK-293 cells mainly when treated with fractions 5-11, whereas fractions $12-18$ and $24-32$ showed only some low-level signal (Fig. 3b). Finally, we analyzed the fractions for protein impurities. Our SDS-PAGE results show that VP1, VP2, and VP3 could be clearly identified in fractions 510 , whereas massive protein contaminations were obtained in fractions 11 and 12 (Fig. 3c). The presence of contaminating proteins was also evident from an increase in absorption at $340 \mathrm{~nm}$ (Supplementary Fig. S3), which was previously 


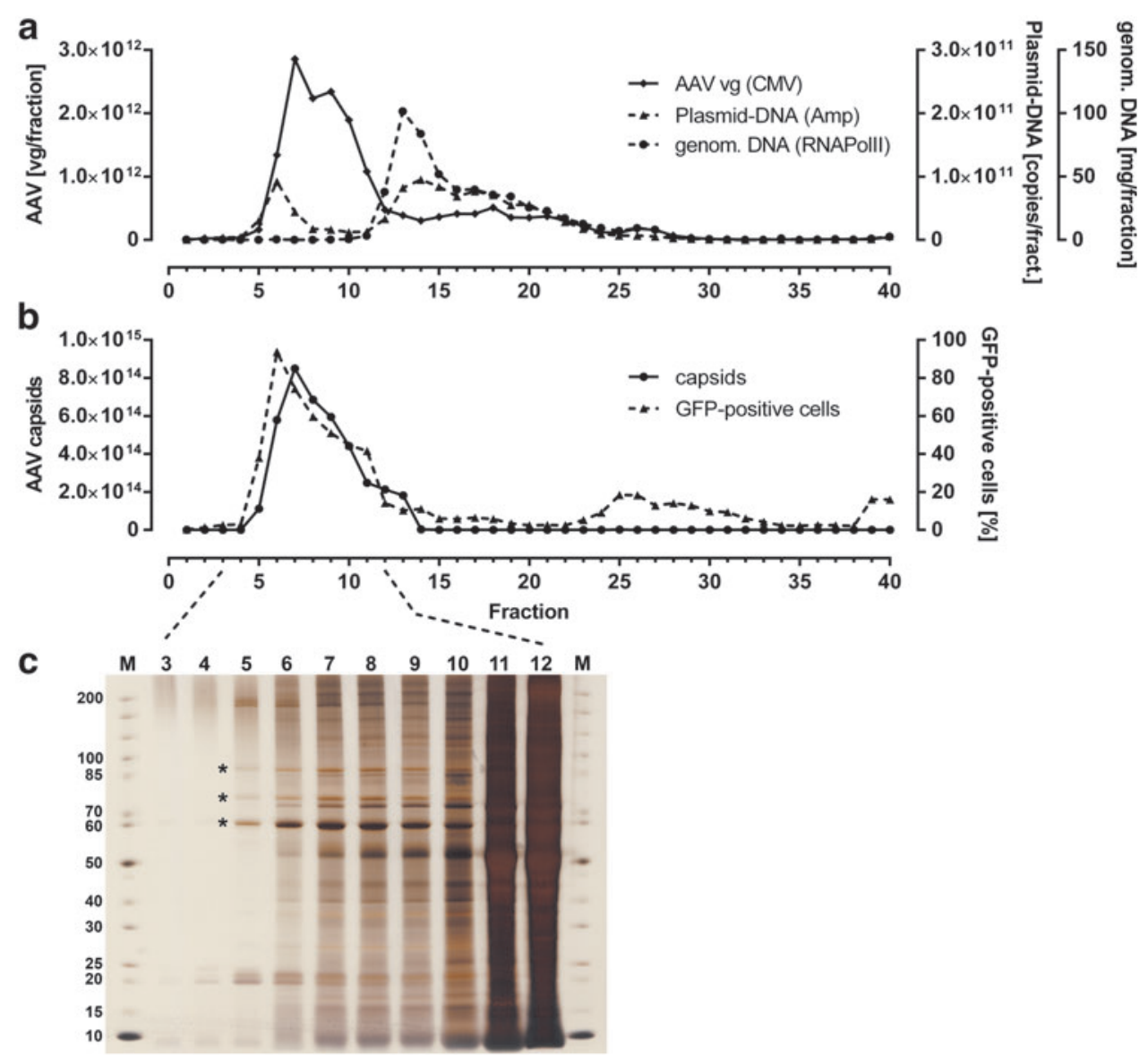

Figure 3. Analysis of the fractionated iodixanol density gradient. One-milliliter fractions were collected from the bottom of the ultracentrifugation tube and analyzed for bioactive AAV particles and contaminants analogous to the description in Fig. 2. Color image available online at www.liebertpub.com/hgtb

shown to be a suitable measuring method to separate AAV-containing fractions from the major protein contaminations in the upper fractions of the $40 \%$ phase. $^{5}$ In addition, a drop in the RI can serve as a further indicator of iodixanol phase changes (Supplementary Fig. S4). Taken together, to obtain bioactive AAV particles with the lowest impurities possible, fractions $6-10$ should be isolated.

\section{Comparative analysis of polishing methods for AAVs isolated from iodixanol gradient}

Despite the fact that iodixanol is a clinically approved contrast agent and has previously been shown to behave inertly in rodents, ${ }^{9,10}$ depletion of iodixanol from AAV preparations is desirable in some instances, for example, for in vivo applications, where the viscosity of iodixanol could be hindering. We therefore compared various methods for their ability to deplete iodixanol and further purify AAVs isolated from the $40 \%$ iodixanol phase. For this purpose, we purified the cell lysate obtained from a $50 \times 15 \mathrm{~cm}$ plate-AAV8-GFP produc- tion batch by two parallel gradients, pooled the AAV target fractions, and quartered them for the subsequent polishing steps using the following methods: (1) ultrafiltration/concentration using Amicon Ultra-15, $100 \mathrm{kDa}$ MWCO centrifugation tubes; (2) size-exclusion chromatography (SEC) using the disposable PD-10 desalting column and subsequent Amicon UItra-15 concentration; (3) hollow-fiber tangential flow ultrafiltration (HFTFF) using the MicroKros hollow-fiber module and a 100-kDa MWCO membrane; and (4) PEG-8000 precipitation, followed by dialysis and concentration with the Amicon Ultra-15 device.

To assess the loss of AAV vectors associated with each of the purification methods, we first conducted qPCR quantification of the final AAV vector preparations relative to the common starting material. The results showed that ultrafiltration reached the highest AAV recovery at $92.6 \%$, followed by SEC at $72.5 \%$, $\mathrm{HF}-\mathrm{TFF}$ at $70.3 \%$, and PEG precipitation at $56.0 \%$ (Fig. 4a). SDS-PAGE analysis revealed that the AAVs purified by ultrafiltration, HF-TFF, and PEG precipitation were similarly clean as opposed to 

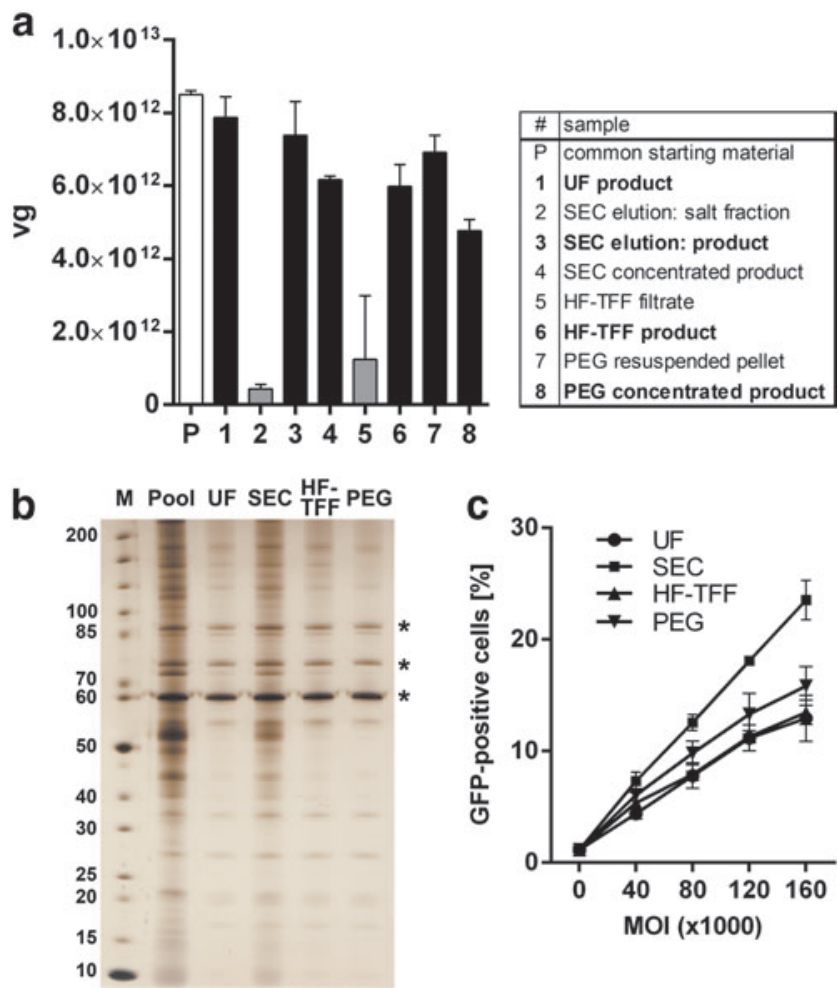

Figure 4. Comparative analysis of polishing methods for AAVs isolated from an iodixanol gradient. AAV target fractions obtained from two parallel iodixanol gradients loaded with the HEK-293 cell lysate from a total of fifty $15-\mathrm{cm}$ plates were further purified by ultrafiltration (UF), size-exclusion chromatography (SEC), hollow-fiber tangential flow filtration (HF-TFF), or PEG-8000 precipitation. (a) AAV vector yield and loss during purification by the various polishing methods as quantified by qPCR (single observations, $n=3$ assay replicates, means \pm SD). (b) Silver-stained SDS-polyacrylamide gel of each of the final AAV preparations relative to the common starting material (pool). A total of $2 \times 10^{9} \mathrm{VG}$ was loaded per lane. M, marker lane. Asterisks indicate VP1, VP2, and VP3. (c) Bioactivity of the purified AAV preparations depending on the polishing method used. GFP-positive cells were detected by flow cytometry $48 \mathrm{hr}$ after transduction at increasing multiplicity of infection (MOI). Single observations, $n=4$ assay replicates, means \pm SD. Color image available online at www.liebertpub.com/hgtb

SEC-purified vectors, which showed a clearly higher amount of protein impurities (Fig. 4b). Moreover, whereas the concentration of residual iodixanol was $<0.5 \%$ in AAV preparations purified by ultrafiltration and PEG precipitation, it was $\sim 3 \%$ in HF-TFFpurified samples and $>5 \%$ in SEC-purified samples, as determined by mass spectrometry. Finally, in vitro bioactivity was assessed by transducing HEK-293 cells, which showed the slightly higher bioactivity of SEC-purified vectors as compared with cells transduced with vectors purified by the other polishing methods, which behaved similarly in this assay (Fig. 4c). Therefore, regarding the required time and efficiency to deplete iodixanol and contaminating proteins, ultrafiltration turned out to be the most efficient method for the final purification of iodixanol-derived AAV vectors.

\section{Direct comparison of CsCl- and iodixanol- based AAV purification}

After having defined the criteria for isolation of bioactive AAV particles with the highest degree of purity from both $\mathrm{CsCl}$ and iodixanol gradients, we finally carried out a side-by-side comparison of both protocols. For this purpose, we halved the cell lysate obtained from forty $15-\mathrm{cm}$ plates of HEK-293 cells and purified one-half by the $\mathrm{CsCl} / \mathrm{UF}$-based protocol and the other half by the iodixanol/UFbased protocol. This approach was repeated for three separately produced AAV batches, each of which was purified independently.

Regarding AAV recovery relative to the common starting material, no significant differences were observed between the CsCl- and iodixanol-based purification protocols (Fig. 5a). However, the io-
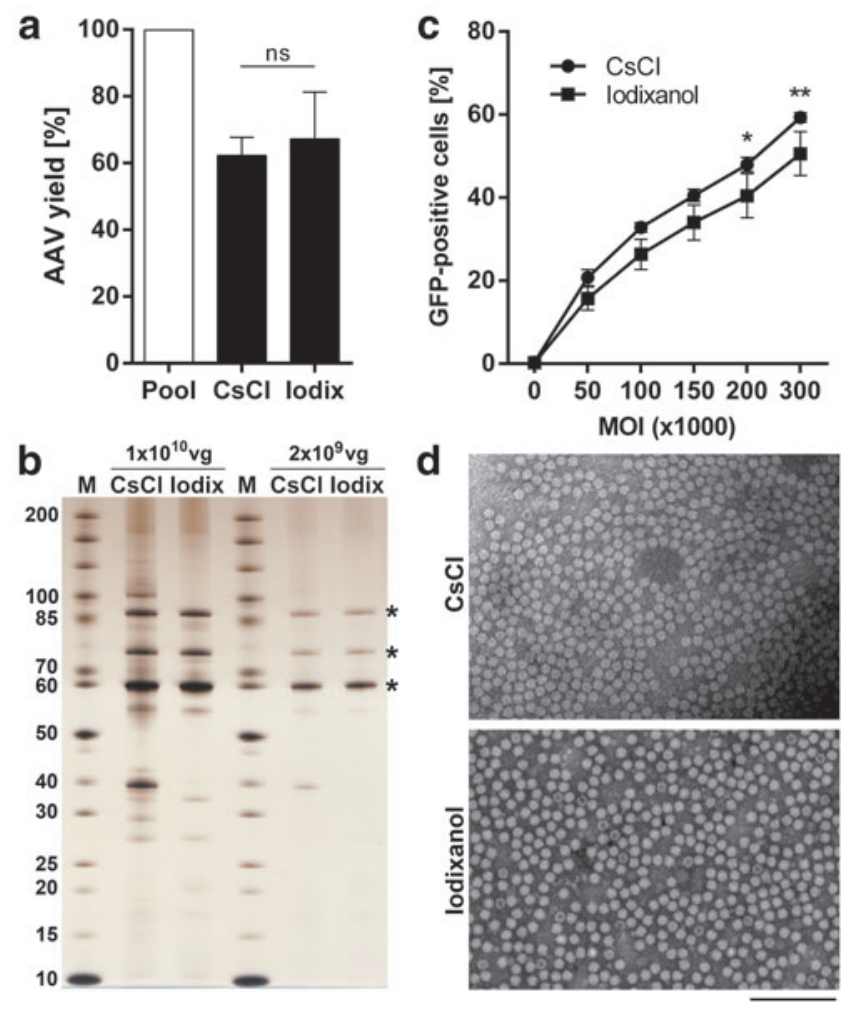

Figure 5. Direct comparison of CsCl- and iodixanol-based AAV purification. A HEK-293 cell lysate of three independently produced AAV batches (forty 15$\mathrm{cm}$ plates each) was halved and purified by either CsCl- or iodixanol-based purification as depicted in Fig. 1. (a) AAV recovery measured by qPCR of the final product relative to the common starting material (pool, set $100 \%$ ). $n=3$ $A A V$ batches, means $\pm S D$. (b) Silver-stained SDS-polyacrylamide gel of the final products of batch 1. For batches 2 and 3, see Supplementary Fig. S5. Either $1 \times 10^{10}$ or $2 \times 10^{9} \mathrm{VG}$ was loaded per lane, as indicated. M, marker lane. Asterisks indicate VP1, VP2, and VP3. (c) Bioactivity of the final AAV preparations. GFP-positive cells were detected by flow cytometry $48 \mathrm{hr}$ after transduction at increasing multiplicities of infection (MOls). $n=3$ AAV batches, means \pm SD. ${ }^{*} p<0.05,{ }^{* *} p<0.01$. (d) Transmission electron microscopy anal$y$ sis of the final AAV preparations of batch 1 (original magnification, $\times 16,000$ ). Scale bar, $200 \mathrm{~nm}$. Color image available online at www.liebertpub.com/hgtb 
dixanol protocol showed fewer protein impurities as evident from SDS-PAGE analysis (Fig. 5b and Supplementary Fig. S5), whereas the CsCl-derived AAVs demonstrated slightly higher in vitro bioactivity on HEK-293 cells (Fig. 5c). Finally, we quantified the amount of full and empty capsids in each preparation, using images obtained by TEM (Fig. 5d). Our results demonstrate that AAV preparations obtained by the iodixanol protocol contained approximately $20 \%$ empty particles, whereas almost no empty particles $(<1 \%)$ were found in $\mathrm{CsCl}-$ derived preparations. In summary, whereas the iodixanol protocol led to AAV vectors of higher purity, the CsCl-purified preparation was slightly more bioactive and had a nearly optimal full-toempty particle ratio.

\section{Analysis of protein impurities in HEK-293- derived AAV product preparations}

Despite the relatively high degree of purity obtained with both CsCl- and iodixanol-based protocols, protein impurities became visible in both cases when higher AAV amounts were analyzed by SDSPAGE (Fig. 5b and Supplementary Fig. S5). Because protein contamination in AAV vector preparations might cause immunological responses in vivo, which could potentially alter relevant experimental readouts, we attempted to identify the most prominent protein contamination in the final AAV preparations. For this purpose, we reran the final samples from our direct comparison of CsCl- and iodixanolderived AAVs (Fig. 5b) on an SDS-polyacrylamide gel. After Coomassie blue staining, the most prominent bands (Fig. 6, indicated by numbers) were excised and analyzed by mass spectrometry. Notably, whereas bands 7-13 were present at similar intensity in CsCl- and iodixanol-purified preparations, bands 1-6 seemed to be present relatively specifically in the less pure $\mathrm{CsCl} \mathrm{AAV} \mathrm{preparations} \mathrm{(Fig.} \mathrm{6).}$ A further point to note is that the band pattern was highly reproducible when comparing three independently produced and purified AAV batches (Supplementary Fig. S6).

As expected, mass spectrometry successfully identified the AAV capsid proteins VP1, VP2, and VP3 in bands 7-9. However, capsid protein fragments were also detected in bands 4 and 10-13. Moreover, besides the identification of known AAVinteracting proteins including the TAF-1/SET complex components protein SET (bands 3 and 4), ANP32A and ANP32B (band 5), as well as nucleolin (band 2) and nucleophosmin (band 4), several other cellular proteins were identified that have not been reported in the context of AAV so far (Table 1). Among these are the heterogeneous nu-

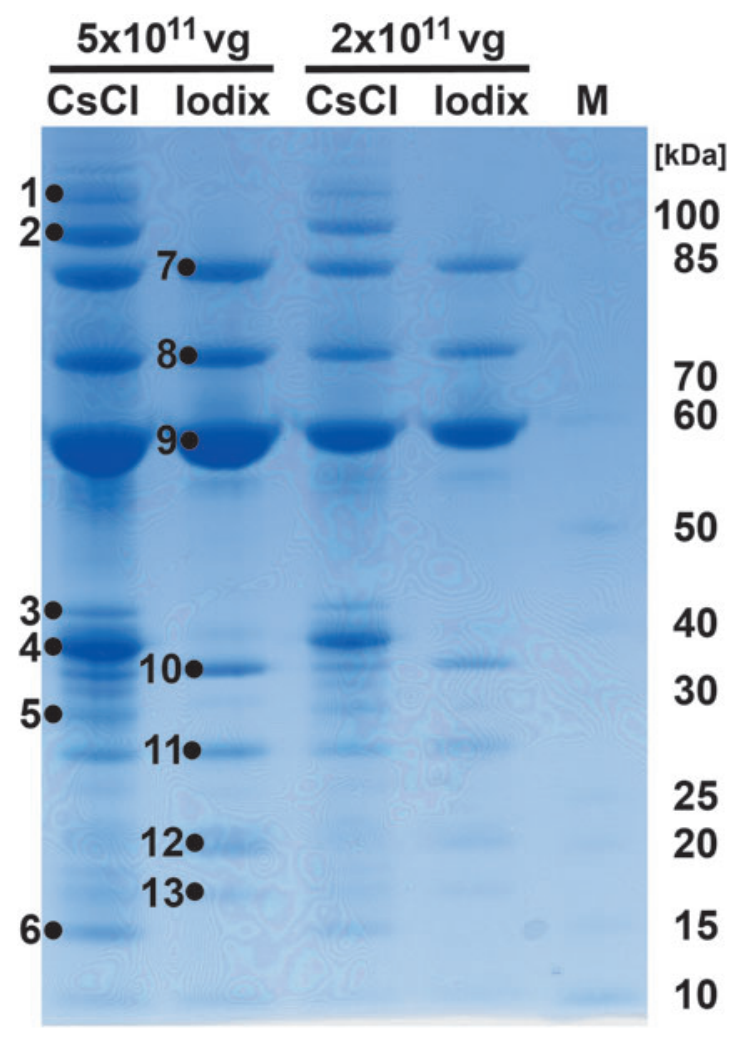

Figure 6. Analysis of protein impurities in HEK-293-derived AAV product preparations. Shown is a Coomassie blue-stained SDS-polyacrylamide gel of each of the final AAV preparations of batch 1 loaded at the indicated amounts. For batches 2 and 3, see Supplementary Fig. S6. Gel bands 1-13 were analyzed by mass spectrometry, and identified cellular proteins are presented in Table 1. M, marker lane. Color image available online at www.liebertpub.com/hgtb

clear ribonucleoproteins HNRNPAB, HNRNPCL1, and HNRNPA2B1 (bands 3-5), the spliceosome proteins SRSF1 and SNRPD3 (bands 5 and 6), the mitochondrial single-stranded DNA-binding protein SSBP1 (band 6), the ferritin heavy and light chain proteins FTH1 and FTL (band 12), and the putative deoxyribose-phosphate aldolase DERA (band 10). These results might suggest that these proteins could interact with AAV at some point during its life cycle.

\section{DISCUSSION}

In our study we systematically compared CsCland iodixanol-based methods for the purification of recombinant AAV vectors. Fractionation experiments provided detailed insight into the distribution of intact AAV particles and unwanted contaminants within the density gradients and allowed the definition of specific criteria for the harvest of bioactive viral vectors. Moreover, we showed that ultrafiltration in a centrifugation device was the most powerful method to further polish iodixanol-purified AAVs. 
Table 1. Cellular Proteins Identified in Adeno-Associated Viral Product Preparations

\begin{tabular}{|c|c|c|}
\hline Band no. & Gene name & Protein name \\
\hline 1 & $\begin{array}{l}\text { HDGFRP2 } \\
\text { HTATSF1 }\end{array}$ & $\begin{array}{l}\text { Hepatoma-derived growth factor-related protein } 2 \\
\text { HIV Tat-specific factor } 1\end{array}$ \\
\hline 2 & $\begin{array}{l}\text { HSP9OB1 } \\
\text { NCL }\end{array}$ & $\begin{array}{l}\text { Endoplasmin } \\
\text { Nucleolin }\end{array}$ \\
\hline 3 & $\begin{array}{l}\text { HNRNPAB } \\
\text { SET }\end{array}$ & $\begin{array}{l}\text { Heterogeneous nuclear ribonucleoprotein A/B } \\
\text { Protein SET }\end{array}$ \\
\hline 4 & $\begin{array}{l}\text { HNRNPCL1 } \\
\text { NPM1 } \\
\text { SET }\end{array}$ & $\begin{array}{l}\text { Heterogeneous nuclear ribonucleoprotein C-like } 1 \\
\text { Nucleophosmin } \\
\text { Protein SET }\end{array}$ \\
\hline 5 & ANP32A & $\begin{array}{l}\text { Acidic leucine-rich nuclear phosphoprotein } \\
32 \text { family member } A\end{array}$ \\
\hline & $A N P 32 B$ & $\begin{array}{l}\text { Acidic leucine-rich nuclear phosphoprotein } \\
32 \text { family member B }\end{array}$ \\
\hline & $\begin{array}{l}\text { HNRNPA2B1 } \\
\text { SRSF1 }\end{array}$ & $\begin{array}{l}\text { Heterogeneous nuclear ribonucleoproteins A2/B1 } \\
\text { Splicing factor, arginine/serine-rich } 1 \text { (SF2) }\end{array}$ \\
\hline 6 & $\begin{array}{l}\text { SNRPD2 } \\
\text { SNRPD3 } \\
\text { SSBP1 }\end{array}$ & $\begin{array}{l}\text { Small nuclear ribonucleoprotein Sm D2 } \\
\text { Small nuclear ribonucleoprotein Sm D3 } \\
\text { Single-stranded DNA-binding protein, mitochondrial }\end{array}$ \\
\hline 7 & ALDH16A1 & Aldehyde dehydrogenase family 16 member $\mathrm{A} 1$ \\
\hline 10 & DERA & Putative deoxyribose-phosphate aldolase \\
\hline 12 & $\begin{array}{l}\text { FTH1 } \\
\text { FTL }\end{array}$ & $\begin{array}{l}\text { Ferritin heavy chain } \\
\text { Ferritin light chain }\end{array}$ \\
\hline
\end{tabular}

Last, we found that iodixanol-purified AAVs showed higher purity but also a higher fraction of empty capsids $(\sim 20 \%)$ as compared with CsCl-derived vectors $(<1 \%$ empty particles). Thus, our study can serve as a guide for choosing which method to use for the purification of recombinant AAV vectors and further provides detailed criteria for the setup of respective protocols.

Our analysis of single fractions of the $\mathrm{CsCl}$ and iodixanol gradients demonstrates that contaminating DNA can be efficiently separated from the AAV target fractions. In fact, most plasmid DNA was found in the same fractions as genomic DNA, which could be expected because of the Benzonase digestion step in both protocols. However, in both gradients a distinct plasmid DNA peak separate from genomic DNA could also be identified that matched the peak of AAV genome copies, which might represent wrongly packaged plasmid backbone, as previously reported. ${ }^{11}$ By comparing the peak values of AAV genome copies and plasmid DNA, a ratio of $1.24 \%$ particles with putatively falsely packaged DNA for the $\mathrm{CsCl}$ gradient and $3.22 \%$ for the iodixanol gradient could be calculated. Although confirmation of this hypothesis would need some deeper investigation, a general strategy to prevent wrong packaging would be to increase the size of the plasmid backbone to more than $5 \mathrm{~kb}$.

By comparing various easily implementable polishing methods for their ability to deplete iodixanol and further purify iodixanol-derived AAVs, ultrafiltration using the Amicon Ultra-15 devices with an
MWCO of $100 \mathrm{kDa}$ turned out to be the most efficient method: $92.6 \%$ of the AAV particles were recovered by this method, and it also efficiently depleted iodixanol from $40 \%$ to less than $0.5 \%$ and reached the highest overall AAV purity. Moreover, ultrafiltration could be completed in about $2 \mathrm{hr}$, which was tolerable when compared with SEC $(\sim 1 \mathrm{hr})$, HFTFF ( $\sim 1 \mathrm{hr})$, and PEG precipitation $(\sim 6 \mathrm{hr})$. Furthermore, whereas additional ultrafiltration had to be applied after SEC and PEG precipitation to concentrate the AAV solutions, ultrafiltration alone allows for purification and concentration in one step, which might explain the high recovery rate observed with this strategy. Interestingly, despite the fact that SEC-purified AAVs retained the highest degree of protein impurities, they showed higher bioactivity in HEK-293 cells as compared with the cleaner AAV preparations obtained by the other methods. One possible explanation for this observation is that the remaining host cell proteins might improve transduction efficiency of AAV vectors in vitro, as previously reported. ${ }^{12}$

Our final side-by-side comparison of CsCl- and iodixanol-based purification demonstrated that the iodixanol protocol was slightly better regarding purity and offers the significant advantage of speed, because purification can be completed within 1 day. However, TEM analysis also revealed that about $20 \%$ of iodixanol-derived AAV preparations consisted of empty particles, whereas empty particles were present in CsCl-purified preparations at less than $1 \%$. As demonstrated in the fractionation experiments, a distinct capsid peak containing the empty particles was detected in fraction 23 in the $\mathrm{CsCl}$ gradient, whereas no such peak, but only a peak shoulder, was present in the iodixanol gradient (fractions 12 and 13). While this "shoulder" likely contains the empty particles, these will hardly be depletable by narrowing the range of pooled fractions without losing a significant amount of intact AAVs. Despite the higher amount of impurities present in the CsCl-derived preparations, these AAVs showed slightly higher bioactivity in vitro than iodixanol-purified vectors. Although these differences were marginal and statistically significant only at the two highest AAV concentrations, they might mirror the fact that fewer receptors are occupied by empty particles, thereby enabling more efficient transduction by full AAVs. Again, it also cannot be ruled out that the higher degree of impurities in CsCl-derived preparations might facilitate more efficient transduction. ${ }^{12}$

Although our finding that iodixanol purification allows for the efficient recovery of highly bioactive vectors that show superior purity as compared with 
CsCl-purified vectors is consistent with the findings of Zolotukhin and colleagues, ${ }^{3}$ we could not confirm the drastic differences between iodixanol and $\mathrm{CsCl}$ regarding bioactivity. In fact, whereas Zolotukhin and colleagues reported CsCl-purified vectors to be much less bioactive than iodixanolpurified vectors, we observed similar bioactivity for both preparations. However, there are also some distinct differences between the two studies, which might not only explain the observed discrepancies but also limit comparability: First, our $\mathrm{CsCl}$ protocol included $\mathrm{CaCl}_{2}$ and PEG precipitation steps before ultracentrifugation, whereas their protocol used ammonium sulfate precipitation. Second, whereas we compared protocols based on a single ultracentrifugation step, the other study used combined iodixanol and affinity chromatography purification, combined iodixanol and $\mathrm{CsCl}$ purification, or protocols based on two rounds of $\mathrm{CsCl}$. Because it is known that extended exposure to $\mathrm{CsCl}$ can reduce AAV bioactivity, ${ }^{9}$ this point might be particularly relevant. Finally, we used HEK-293 cell transduction to assess bioactivity, whereas the other study assessed AAV infectivity and replication using assays that rely on adenovirus coinfection.

In addition to the findings reported here, we could not observe any drastic purification methoddependent differences in vector bioactivity in a large number of mouse studies conducted in our laboratory, where both iodixanol- and CsCl-purified vectors were used, thereby suggesting that both methods are well suited for use in research studies. Yet, systematic in vivo comparisons should be conducted to analyze potential differences in detail, especially with respect to capsid-dependent immunogenicity.

Finally, our proteomics approach revealed interesting insight into the identity of protein impurities present in the AAV vector preparations. The identification of nucleolin, nucleophosmin, and SSBP1 confirms previous studies that demonstrated interaction of these proteins with the AAV capsid. ${ }^{13-15}$ Our finding that protein SET as well as ANP32A and ANP32B were present in the AAV product further underscores the importance of the TAF-1/SET/ ANP32A/B complex for AAV DNA replication. ${ }^{16}$ Of the other identified proteins, to our knowledge none has been described in the context of AAV so far. However, heterogeneous ribonucleoproteins including HNRNPA2B1 and HNRNPAB were shown to interact with the capsid of feline parvovirus ${ }^{17}$ and to be involved in viral replication and assembly of human papillomavirus (HPV), HIV, mouse hepatitis virus (MHV), Japanese encephalitis virus (JEV), and Junin virus. ${ }^{18-22}$ Moreover, HTATSF1 and HSP90B1 have been linked to HIV transcription, ${ }^{23}$ splicing, ${ }^{24}$ and infectivity, ${ }^{25}$ respectively. We further identified the spliceosome-associated proteins SNRPD2/3 and SRSF1 (also known as ASF/SF2), the latter of which was found to be involved in hepatitis delta virus (HDV), HIV, and adenovirus replication. ${ }^{26-28}$ Finally, for the other proteins identified we did not find any studies describing involvement in virus biology.

Interestingly, whereas some protein bands (e.g., bands 10-13) were similarly intense in both CsCland iodixanol-purified preparations, others (bands 1-4) showed much higher intensity in CsCl-derived product, suggesting that the respective proteins (e.g., nucleolin, SET) are more efficiently depleted by iodixanol purification. Although we did not systematically analyze all equally sized bands of both products (i.e., $\mathrm{CsCl}$ and iodixanol derived), we identified several proteins in both preparations, among these the ferritin heavy (FTH1) and light chain (FTL), SNRPD3, and the putative deoxyribosephosphate aldolase DERA, thereby supporting the hypothesis that these proteins may interact with AAV at some point during its life cycle. Although our results represent an interesting starting point, further studies are needed to explore the exact role of these proteins and whether these are specific to AAV8 or independent of the capsid variant used. Respective studies should further help to clarify whether copurification of these proteins is a random event or indeed due to specific interaction with AAV.

In conclusion, our study provides a comprehensive insight into CsCl- and iodixanol-based protocols for AAV purification, which will serve as a helpful guide for the decision on which protocol to use and, further, for the setup of respective purification protocols, especially in laboratories newly entering the AAV field.

\section{ACKNOWLEDGMENTS}

The authors thank Kai Zuckschwerdt for excellent technical assistance. The authors are further indebted to Dagmar Rädel and Martin Dass for carrying out electron microscopy. Last, the authors thank Tanja Scheffold for supporting mass spectrometry analysis.

\section{AUTHOR DISCLOSURE}

All authors listed are employees and receive salary from Boehringer Ingelheim Pharma $\mathrm{GmbH}$ $\&$ Co. KG. 


\section{REFERENCES}

1. Strobel B, Duechs MJ, Schmid R, et al. Modeling pulmonary disease pathways using recombinant adeno-associated virus 6.2. Am J Respir Cell Mol Biol 2015 (in press).

2. Grimm D, Pandey K, and Kay MA. Adenoassociated virus vectors for short hairpin RNA expression. Methods Enzymol 2005;392:381-405.

3. Zolotukhin S, Byrne BJ, Mason E, et al. Recombinant adeno-associated virus purification using novel methods improves infectious titer and yield. Gene Ther 1999;6:973-985.

4. Klein RL, Dayton RD, Tatom JB, et al. AAV8, 9, Rh10, Rh43 vector gene transfer in the rat brain: effects of serotype, promoter and purification method. Mol Ther 2008;16:89-96.

5. Lock M, Alvira M, Vandenberghe LH, et al. Rapid, simple, and versatile manufacturing of recombinant adeno-associated viral vectors at scale. Hum Gene Ther 2010;21:1259-1271.

6. Shevchenko A, Wilm M, Vorm 0 , et al. Mass spectrometric sequencing of proteins silver-stained polyacrylamide gels. Anal Chem 1996;68: 850-858.

7. Helm D, Vissers JPC, Hughes CJ, et al. Ion mobility tandem mass spectrometry enhances performance of bottom-up proteomics. Mol Cell Proteomics 2014;13: 3709-3715.

8. Biemann K. Sequencing of peptides by tandem mass spectrometry and high-energy collision-induced dissociation. Methods Enzymol 1990;193: 455-479.

9. Hermens WT, ter Brake 0, Dijkhuizen PA, et al. Purification of recombinant adeno-associated virus by iodixanol gradient ultracentrifugation allows rapid and reproducible preparation of vector stocks for gene transfer in the nervous system. Hum Gene Ther 1999;10:1885-1891.

10. Larsen LE, Heglund IF, Fabian R, et al. Neural tolerability of iodixanol in mice and dogs after single and repeated intracisternal administration. Acta Radiol Suppl 1995;399:238-243.
11. Chadeuf G, Ciron C, Moullier P, et al. Evidence for encapsidation of prokaryotic sequences during recombinant adeno-associated virus production and their in vivo persistence after vector delivery. Mol Ther 2005;12:744-753.

12. Tenenbaum L, Hamdane M, Pouzet $M$, et al. Cellular contaminants of adeno-associated virus vector stocks can enhance transduction. Gene Ther 1999;6:1045-1053.

13. Qiu J, and Brown KE. A 110-kDa nuclear shuttle protein, nucleolin, specifically binds to adenoassociated virus type 2 (AAV-2) capsid. Virology 1999;257:373-382.

14. Bevington JM, Needham PG, Verrill KC, et al. Adenoassociated virus interactions with B23/Nucleophosmin: identification of sub-nucleolar virion regions. Virology 2007;357:102-113.

15. Dong B, Duan X, Chow HY, et al. Proteomics analysis of co-purifying cellular proteins associated with rAAV vectors. PLoS One 2014;9:e86453.

16. Pegoraro G, Marcello A, Myers MP, et al. Regulation of adeno-associated virus DNA replication by the cellular TAF-I/set complex. J Virol 2006:80:6855-6864.

17. Wang D, and Parrish CR. A heterogeneous nuclear ribonucleoprotein $\mathrm{A} / \mathrm{B}$-related protein binds to single-stranded DNA near the $5^{\prime}$ end or within the genome of feline parvovirus and can modify virus replication. J Virol 1999;73:7761-7768.

18. Li X, Johansson C, Glahder J, et al. Suppression of HPV-16 late L1 5'-splice site SD3632 by binding of hnRNP D proteins and hnRNP A2/B1 to upstream AUAGUA RNA motifs. Nucleic Acids Res 2013;41:10488-10508.

19. Lévesque K, Halvorsen M, Abrahamyan L, et al. Trafficking of HIV-1 RNA is mediated by heterogeneous nuclear ribonucleoprotein A2 expression and impacts on viral assembly. Traffic 2006;7:1177-1193.

20. Shi ST, Yu G-Y, and Lai MM. Multiple type A/B heterogeneous nuclear ribonucleoproteins (hnRNPs) can replace hnRNP A1 in mouse hepatitis virus RNA synthesis. J Virol 2003;77:10584-10593.
21. Katoh H, Mori $Y$, Kambara $H$, et al. Heterogeneous nuclear ribonucleoprotein A2 participates in the replication of Japanese encephalitis virus through an interaction with viral proteins and RNA. J Virol 2011;85:10976-10988.

22. Maeto CA, Knott ME, Linero FN, et al. Differential effect of acute and persistent Junin virus infections on the nucleo-cytoplasmic trafficking and expression of heterogeneous nuclear ribonucleoproteins type A and B. J Gen Virol 2011;92:2181-2190.

23. Green VA, Arbuthnot $P$, and Weinberg MS. Impact of sustained RNAi-mediated suppression of cellular cofactor Tat-SF1 on HIV-1 replication in CD4 ${ }^{+}$ T cells. Virol J 2012;9:272.

24. Miller HB, Saunders KO, Tomaras GD, et al. TatSF1 is not required for Tat transactivation but does regulate the relative levels of unspliced and spliced HIV-1 RNAs. PLoS One 2009;4:e5710.

25. Landi A, Vermeire J, lannucci V, et al. Genomewide shRNA screening identifies host factors involved in early endocytic events for HIV-1-induced CD4 down-regulation. Retrovirology 2014;11:118.

26. Sikora D, Greco-Stewart VS, Miron P, et al. The hepatitis delta virus RNA genome interacts with eEF1A1, p54 ${ }^{\text {nrb }}$, hnRNP-L, GAPDH and ASF/SF2. Virology 2009;390:71-78.

27. Maertens GN, Cook NJ, Wang W, et al. Structural basis for nuclear import of splicing factors by human Transportin 3. Proc Natl Acad Sci U S A 2014;111: 2728-2733.

28. Mui MZ, Kucharski M, Miron M-J, et al. Identification of the adenovirus E4orf4 protein binding site on the $B 55 \alpha$ and $\mathrm{Cdc} 55$ regulatory subunits of PP2A: implications for PP2A function, tumor cell killing and viral replication. PLoS Pathog 2013;9:e1003742.

Received for publication July 21, 2015; accepted after revision July 23, 2015.

Published online: July 28, 2015. 